\title{
Annealing-free fabrication of highly oxidation-resistive copper nanowire composite conductors for photovoltaics
}

\begin{abstract}
Yulim Won ${ }^{1}$, Areum Kim ${ }^{1}$, Donggyu Lee ${ }^{1}$, Wooseok Yang${ }^{1}$, Kyoohee Woo ${ }^{1}$, Sunho Jeong ${ }^{2}$ and Jooho Moon ${ }^{1}$
Copper nanowire (CuNW)-network film is a promising alternative to the conventional indium tin oxide (ITO) as a transparent conductor. However, thermal instability and the ease of oxidation hinder the practical applications of CuNW films. We present oxidation-resistive CuNW-based composite electrodes that are highly transparent, conductive and flexible. Lactic acid treatment effectively removes both the organic capping molecule and the surface oxide/hydroxide from the CuNWs, allowing direct contact between the nanowires. This chemical approach enables the fabrication of transparent electrodes with excellent properties $\left(19.8 \Omega \mathrm{sq}^{-1}\right.$ and $88.7 \%$ at $\left.550 \mathrm{~nm}\right)$ at room temperature without any atmospheric control. Furthermore, the embedded structure of CuNWs with Al-doped ZnO (AZO) dramatically improves the thermal stability and oxidation resistance of CuNWs. These AZO/CuNW/AZO composite electrodes exhibit high transparency $(83.9 \%$ at $550 \mathrm{~nm}$ ) and low sheet resistance $\left(35.9 \Omega \mathrm{sq}^{-1}\right)$, maintaining these properties even with a bending number of 1280 under a bending radius of $2.5 \mathrm{~mm}$. When implemented in a $\mathrm{Cu}\left(\mathrm{In}_{1-x}, \mathrm{Gax}\right)(\mathrm{S}, \mathrm{Se})_{2}$ thin-film solar cell, this composite electrode demonstrated substantial potential as a low-cost (Ag-, In-free), high performance transparent electrode, comparable to a conventional sputtered ITO-based solar cell. NPG Asia Materials (2014) 6, e105; doi:10.1038/am.2014.36; published online 13 June 2014
\end{abstract}

Keywords: chemical reduction treatment; copper nanowire; indium-free transparent electrodes; photovoltaic; thermal oxidation resistance

\section{INTRODUCTION}

Transparent conductive materials are a crucial, basic element in the realization of various optoelectronic devices, such as flat panel displays, touch screens, organic light emitting diodes and thin-film solar cells. ${ }^{1-3}$ Indium tin oxide (ITO) has been the most widely explored material, because of its excellent transparency ( $\sim 90 \%$ at $550 \mathrm{~nm})$ and low sheet resistance $\left(\sim 20 \Omega \mathrm{sq}^{-1}\right)$. However, with the rising demand for transparent electrodes, the development of cost-effective alternatives to ITO has been of great importance. The quest for alternative transparent conductors, including conducting polymers, carbon nanotubes, graphenes and nanostructured electrodes, has been a topic of active research for the last decade. ${ }^{4}$ Among these materials, metal-nanowire network films are thought to be a substantial candidate. The metallic nanowire film is capable of providing high transparency and conductivity by virtue of its characteristic structure, which consists of a percolated random network of nanowires. ${ }^{5-9}$ Unlike the brittle metal oxide skeletonbased ITO, metal-nanowire films are easily applicable to bendable, wearable active devices, because of the inherently flexible nature of metals. A high aspect ratio is a critical factor for high transparency and conductivity, but metal nanowires possessing this quality suffer from thermal instability. The dramatically lowered melting point of nanostructured, one-dimensional metal materials results in the collapse of the interconnected web-like structure above $200^{\circ} \mathrm{C}$, significantly raising the sheet resistance. To improve thermal stability, metal oxide/nanowire composite electrodes have been exploited, and have been shown to possess optical/electrical properties comparable to ITO as well as superior flexibility. ${ }^{10,11}$ The silver nanowire (AgNWs) sandwiched within $\mathrm{ZnO}$ layers maintained a sheet resistance $\left(R_{\mathrm{sh}}\right)$ around $11.3 \Omega \mathrm{sq}^{-1}$ at $225^{\circ} \mathrm{C}$, while the $R_{\mathrm{sh}}$ of AgNW films soared to $505.5 \Omega \mathrm{sq}^{-1}$ at the same temperature. ${ }^{12}$ However, in spite of the successful demonstration of improved thermal stability of AgNWs, the high cost of noble metals has hindered the wider use of this appealing approach.

Copper is a promising alternative material, as it is highly conductive, but significantly cheaper than Ag. Recently, the development of copper nanowire (CuNW)-based network films has emerged as a new cost-effective, substantial pathway for creating transparent electrodes. ${ }^{13} \mathrm{CuNW}$ network films have been prepared by either electrospinning or solution deposition using nanowire dispersions. Polymer nanofiber webs containing a copper salt were electrospun onto substrates, and the subsequent calcination process, followed by a

${ }^{1}$ Department of Materials Science and Engineering, Yonsei University, Seoul, Republic of Korea and ${ }^{2}$ Advanced Materials Division, Korea Research Institute of Chemical Technology, Daejeon, Republic of Korea

Correspondence: Professor J Moon, Department of Materials Science and Engineering, Yonsei University, 50 Yonsei-ro, Seodaemun-gu, Seoul 120-749, Republic of Korea. E-mail: jmoon@yonsei.ac.kr

Received 5 January 2014; revised 28 March 2014; accepted 7 April 2014 
reduction under $\mathrm{H}_{2}$, led to the formation of copper nanofibers with good electrical resistance $\left(\sim 50 \Omega \mathrm{sq}^{-1}\right)$ and optical transmittance ( $\sim 90 \%$ at $550 \mathrm{~nm}) .{ }^{9}$ The other approach involves CuNW inks, with which transparent electrodes were fabricated by either a coating or filtration method. Large-scale, low-cost fabrication of transparent electrodes could be effectively implemented using this method, as long as high-quality CuNWs are obtainable. The synthesis of CuNWs relies primarily on the chemical reduction of copper precursors in an aqueous medium. CuNWs $\sim 20 \mathrm{~nm}$ in diameter and $\sim 60 \mu \mathrm{m}$ in length were synthesized by either hydrazine or glucose-based reduction of copper salts with the presence of polyvinylpyrrolidone $^{14}$ or hexadecylamine (HDA), ${ }^{15}$ respectively. The resulting nanowire network films exhibited excellent sheet resistance $\left(\sim 30 \Omega \mathrm{sq}^{-1}\right)$ and optical transmittance $(\sim 85 \%$ at $550 \mathrm{~nm})$ as well flexibility. Recently, Zhang et al. ${ }^{16}$ reported a nonaqueous synthetic methodology for ultralong single-crystalline CuNWs.

In addition to thermally induced structural evolution into discontinued, isolated morphologies, the thermal oxidation of copper nanomaterials of high surface area, even at slightly elevated temperatures, should be resolved for CuNW-based network films. Furthermore, in synthesizing CuNWs, the formation of a surface oxide layer should be suppressed in a controlled, sophisticated manner, as the oxide phases are thermodynamically stable; the presence of surface copper oxides increases the annealing temperature for the formation of intrananowire junctions as well as deteriorates the electrical conductivity of CuNW network films. To address the thermal-stability issue, which involves both thermal oxidation and structural evolution, two approaches were proposed: (i) core-shell structure and (ii) lamination-based layered structure. These methods prevent the CuNW surface from being exposed to oxygen by forming a passivation layer. Rathmell et al. ${ }^{17}$ reported the synthesis of the core-shell CuNWs, in which the core CuNWs were surrounded by a nickel shell in the form of cupronickel nanowires. However, the resistivity of such film was 3.1 times higher than pure CuNWs, due to the intrinsically higher resistivity of nickel. Luo et al. ${ }^{18}$ demonstrated the synthesis of the CuNWs surrounded by a silver shell (AgCuNWs), but silver is an expensive metal. The surface passivation of electrospun copper nanofibers with double layers of transparent aluminum-doped zinc oxide (AZO) $/ \mathrm{Al}_{2} \mathrm{O}_{3}$ has been performed by atomic layer deposition, yielding a sheet resistance of $47.1 \Omega \mathrm{sq}^{-1}$ and transmittance of $80 \%$ at $550 \mathrm{~nm} .{ }^{19}$ However, in this methodology, even if the external oxide layers allow for oxidative resistance by preventing the direct contact of copper surfaces with oxygen, the electrospun copper nanofiber films require a high-temperature $\left(500^{\circ} \mathrm{C}\right)$ annealing under a reductive atmosphere. This annealing decomposes the organic residues and allows them to be transformed into the metallic copper phase, which is not amenable to flexible substrates. For lamination-based layered nanowire films by hybridization with the reduced graphene oxide, it is difficult to fulfill a uniform large-area lamination and, inevitably, a reductive post annealing at $180^{\circ} \mathrm{C}$ is required. ${ }^{20}$ Most importantly, as of yet, the chemical and/or physical methodology needed to form surface oxide-free CuNWs for further improvement of their electrical conductivity has not been studied.

\section{EXPERIMENTAL PROCEDURES}

\section{Materials}

Copper (II) chloride dihydrate, 1-HDA, dextrose and DL-lactic acid were purchased from Sigma-Aldrich (St Louis, Missouri, USA). Isopropyl alcohol (IPA) and n-hexane were purchased from Duksan Pure Chemical (Ansan-si, Gyeonggi-do, Korea) and mixed cellulose ester membrane filter (a white filter with a diameter of $25 \mathrm{~mm}$ and a pore size of $1 \mu \mathrm{m}$ in diameter) was purchased from ADVANTEC (Bunkyo-ku, Tokyo, Otowa, Japan). All chemicals were used as received without further purification.

\section{CuNW synthesis and lactic acid treatment}

The CuNWs were synthesized in a manner similar to that previously reported after a slight modificagtion. ${ }^{15}$ To synthesize thin and long CuNWs, $\mathrm{CuCl}_{2} \cdot 2 \mathrm{H}_{2} \mathrm{O}(50 \mathrm{mg}), \mathrm{HDA}(280 \mathrm{mg})$ and dextrose $(100 \mathrm{mg})$ were dissolved in water $(20 \mathrm{ml})$ at room temperature. The final solution was placed in a vial and was aged at room temperature for $12 \mathrm{~h}$ while magnetically stirred. After the vial was capped with paraffin tape, the capped vial was heated in an oil bath at $100^{\circ} \mathrm{C}$ for $6 \mathrm{~h}$ under magnetic stirring. Upon complete reaction, the solution had a red-brown color. The synthesized nanowires were washed three times with hexane and IPA, and then purified CuNWs were stored in IPA. After the nanowire aggregation sediment was removed by centrifugation (500 r.p.m. for $3 \mathrm{~min}$ ), the CuNW dispersion was treated with lactic acid for $10 \mathrm{~s}$. Lactic acid was added into the solution to assist with the removal of residual organics and copper oxide.

\section{Determination of the concentration of the CuNW dispersion}

The concentration of the CuNW dispersion was determined by inductively coupled plasma optical emission spectrometry (OPTIMA 8300, Perkin Elmer, Waltham, MA, USA) analysis. To determine the concentration of the CuNW dispersion used for filtration, a set volume of the dispersion $(3 \mathrm{ml})$ was dissolved in nitric acid $(3 \mathrm{ml})$ followed by evaporation at $70^{\circ} \mathrm{C}$. Then the dried substance was further redissolved in a mixture of deionized water $(1 \mathrm{ml})$, nitric acid $(3 \mathrm{ml})$ and hydrogen peroxide $(1 \mathrm{ml})$.

\section{Preparation of transparent electrodes}

CuNW film. To prepare a transparent nanowire electrode with acid-treated CuNWs, vacuum filtration was used, which involves the filtration of a CuNW suspension containing $1 \mathrm{wt} \%$ of lactic acid through a mixed cellulose ester membrane with an average pore size of $1 \mu \mathrm{m}$. The nanowire density of the film was varied by filtrate volume. The membranes with captured CuNWs were transferred by pressing against the substrate surface with the CuNWs side in contact with the substrate. The membrane was then removed and final CuNW films were rinsed with an acetone followed by vacuum drying at $25^{\circ} \mathrm{C}$.

AZO layer. The AZO bottom layers in the AZO/CuNW/AZO composite electrodes with various $\mathrm{CuNW}$ densities were fabricated by radio frequency (RF) magnetron sputtering. The bottom layer $(\sim 55 \mathrm{~nm})$ was sputtered using an Al-doped $\mathrm{ZnO}$ target $(2 \mathrm{wt} \%)$ at a constant RF power of $100 \mathrm{~W}$, an Argon gas flow of $50 \mathrm{ml}$ and $1 \mathrm{mTorr}$ working pressure at room temperature. AZO films were used without a post annealing under a reduction gas. The CuNW layer was transferred onto the bottom AZO-coated glass substrate. The outermost AZO layer $(25-110 \mathrm{~nm})$ was then deposited on the CuNW/AZO layers by sputtering as described previously.

AgNW film. The AgNW density was controlled by the rotation speed used for the AgNW dispersion (AgNW-25, Seashell Technology, CA, USA). The AgNW films were dried at $150{ }^{\circ} \mathrm{C}$ for $60 \mathrm{~s}$. A ultraviolet ozone treatment was also performed to remove any organic binders and additives from the AgNW film.

\section{Film characterization}

Field emission scanning electron microscopy (JEOL-6701F, JEOL Inc., Peabody, MA, USA) and high-resolution transmission electron microscopy (HRTEM, JEM-ARM200F, JEOL Inc.) with an energy dispersive X-ray spectroscopy (Quantax 400, Bruker Optics Inc., Billerica, MA, USA) were used to characterize the structural properties of the nanostructured thin films. The cross-section of the AZO/CuNW/AZO was achieved using a focused dual ion beam system (Helios NanoLab, Hillsboro, OR, USA) by FEI. The effect of lactic acid treatment was also investigated by Fourier-transform infrared spectroscopy (FT-IR, Vertex70, Bruker Optics Inc.), X-ray diffraction (Ultima IV, RIGAKU, Tokyo, Japan), and X-ray photoelectron spectroscopy-depth profile (XPS-depth profile, monochromated Al K-alpha, Thermo Fisher Scientific Inc., Waltham, MA, USA). The sputtering of an area of $2 \times 2 \mathrm{~mm}^{2}$ was performed with an $\mathrm{Ar}^{+}$ion gun $(0.2 \mathrm{kV})$. All binding energies were 
readjusted with respect to $\mathrm{C} 1 \mathrm{~s}(284.8 \mathrm{eV})$. Optical transmittances $(T)$ were measured using a ultraviolet-visible spectrophotometer (V-630, JASCO, Tokyo, Japan) in the wavelength range from $300-1100 \mathrm{~nm}$. The baseline on the ultraviolet-visible spectrophotometer was corrected using a clear slide glass, free of any nanowires. The sheet resistances $\left(R_{\mathrm{sh}}\right)$ of the CuNW and AZO/ CuNW/AZO composite samples were measured by a four-point probe system (RS8, BEGA Technologies, Gyeonggi-do, Korea) as a function of nanowire density. The resistance reported here is the average value obtained after at least 10 multiple measurements. Thermal oxidation stability testing was also carried out by monitoring the change of the sheet resistance of the AZO/CuNW/AZO composite and the bare CuNW film as a function of the atmospheric temperature. The mechanical flexibility of the CuNW and ITO films as function of the number of bend cycles were also determined by repeatedly bending the composite films with a two-point bending device that consisted of two parallel plates, where the radius of curvature was set at $2.5 \mathrm{~mm}$ and the bend speed was about one cycle per second. For the bending test, the CuNW films on the polyethylene terephthalate substrate were annealed at $180^{\circ} \mathrm{C}$, for $30 \mathrm{~min}$ in nitrogen to facilitate firm contact between the CuNWs before the measurement. The sheet resistance values were measured on the flattened film after a given number of bending cycles by a four-point probe.

\section{Thin-film solar cell fabrication}

The same CIGSSe absorbers produced by co-sputtering (Samsung SDI, Gyeonggi-do, Korea) were used for solar cells fabrication to compare the influence of different transparent top electrodes. A ZnS buffer layer of $\sim 60 \mathrm{~nm}$ was deposited on the CIGSSe by chemical bath deposition. The AZO/CuNW/ AZO composite electrode was fabricated on the $\mathrm{ZnS} / \mathrm{CIGSSe} / \mathrm{Mo} /$ glass as described above. For the reference window layer, an intrinsic (i) $\mathrm{ZnO}$ layer with a thickness of $50 \mathrm{~nm}$ was deposited by RF sputtering at a constant RF power of $60 \mathrm{~W}$ at room temperature, gas flow ratio of Ar: $\mathrm{O}_{2}=50 \mathrm{ml}: 5 \mathrm{ml}$ under a working pressure of $1 \mathrm{mTorr}$. After the deposition of the $i$ - $\mathrm{ZnO}$ layer, an ITO layer with a thickness of $200 \mathrm{~nm}$ was prepared by RF magnetron sputtering (RF power of $100 \mathrm{~W}$ at $200^{\circ} \mathrm{C}$, Ar flow rate of $50 \mathrm{ml}$, and working pressure of $1 \mathrm{mTorr})$. The solar cell containing only the CuNW film as a transparent electrode was also fabricated by vacuum filtration of the CuNW dispersion on the $i$-ZnO/ZnS/CIGSSe/Mo/glass substrate. After then, the samples $\left(2 \times 2.5 \mathrm{~cm}^{2}\right)$ were mechanically scribed into the cells with a total area of $0.15 \mathrm{~cm}^{2}$. Finally, silver front contacts were made on the window electrodes as a dot shape (diameter $=1 \mathrm{~mm}$ ) by silver paste. The photovoltaic performance in terms of the $J-V$ of the devices was determined by means of a solar simulator (Sol3A Class AAA, Oriel Instruments, Stratford, CT, USA) and a Keithley (Cleveland, OH, USA) 2400 source measurement unit carried out under air mass 1.5 and 1 sun $\left(100 \mathrm{~mW} \mathrm{~cm}^{-2}\right)$ conditions. The 1 sun intensity level was calibrated using a standard Si reference cell certified by the Newport Corporation (Irvine, CA, USA). From the measured $J_{S C}, V_{O C}$ and $F F$ values, power conversion efficiency (PCE) values were calculated by applying PCE $=$ $F F \times V_{O C} \times J_{S C} / P_{s}$, where $P_{s}$ is the input solar irradiance $\left(\mathrm{mW} \mathrm{cm}^{-2}\right)$. The $F F$ is defined as $F F=J_{m} V_{m} / J_{S C} V_{O C}$, where $J_{m}$ and $V_{m}$ are the maximum current and voltage, respectively.

\section{RESULTS AND DISCUSSION}

Herein, we report highly stable CuNW-based transparent composite electrodes with excellent transparency and conductivity. The CuNW network films were prepared using CuNWs, the surface oxide of which was easily etched by the heretofore-unrecognized carboxylic acid treatment at a room temperature without any atmospheric control. The carboxylic acid treatment effectively removed the organic capping molecules as well as the surface oxide/hydroxide, which significantly reduced the junction resistance of the metal-nanowire contacts without a high temperature post annealing. The subsequent passivation of chemically treated CuNWs with a transparent conductive oxide (for example, AZO) provided excellent long-term thermal stability in air at elevated temperatures. Our approach enables the fabrication of a transparent AZO/CuNW/AZO composite electrode at room temperature, which possessed excellent transparency and low sheet resistance with excellent flexibility. Also presented is an evaluation of this new material-based, layer-structured composite electrode as a transparent conductor, demonstrating applicability to thin-film solar cells.

CuNWs were synthesized according to the method suggested by Jin et al., ${ }^{15}$ with slight modifications (see Supplementary Information for details). As-synthesized CuNWs, with an average diameter $\sim 66 \pm 17 \mathrm{~nm}$ and average length $>50 \mu \mathrm{m}$, were dispersed in IPA with a concentration of $0.06 \mathrm{mg} \mathrm{ml}^{-1}$. The transparent CuNW network films were prepared by a vacuum filtration method, followed by transfer printing onto a slide glass. The as-prepared network film was highly resistive, yielding a sheet resistance of nearly $150 \Omega \mathrm{sq}^{-1}$.
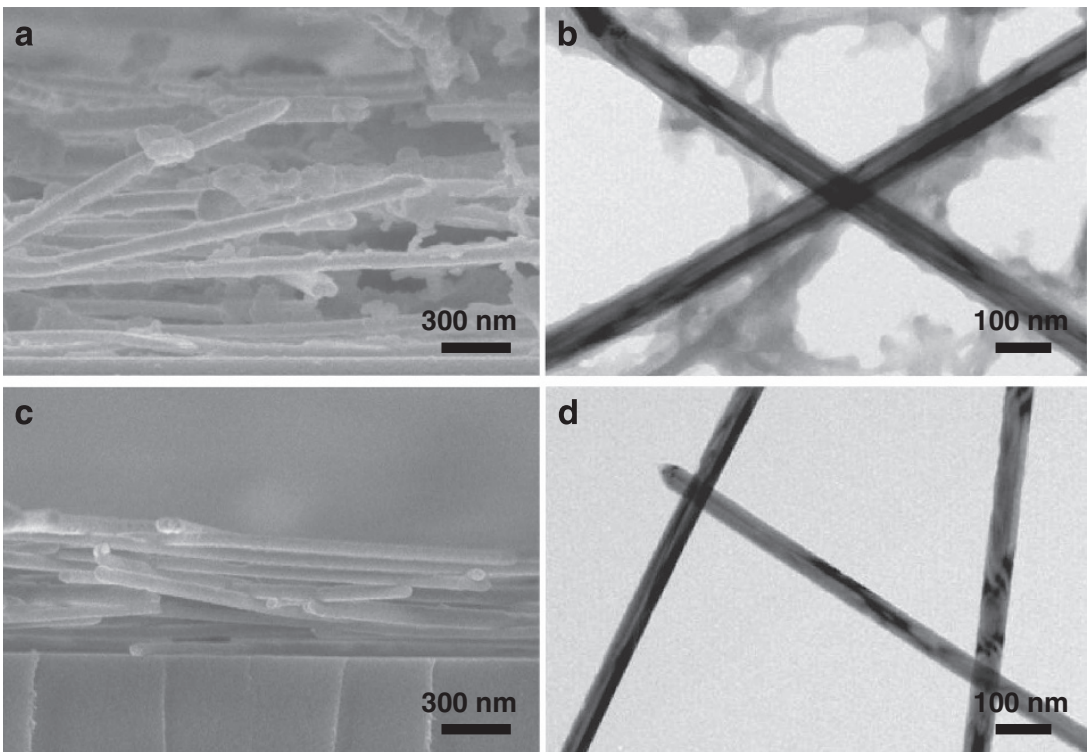

Figure 1 Surface characterizations of copper nanowire. (a) Field emission scanning electron microscopy (FESEM) cross-sectional image and (b) high-resolution transmission electron microscopy (HRTEM) image of as-synthesized CuNW film. (c) FESEM cross-sectional image and (d) HRTEM image of lactic acid-treated CuNW film. 


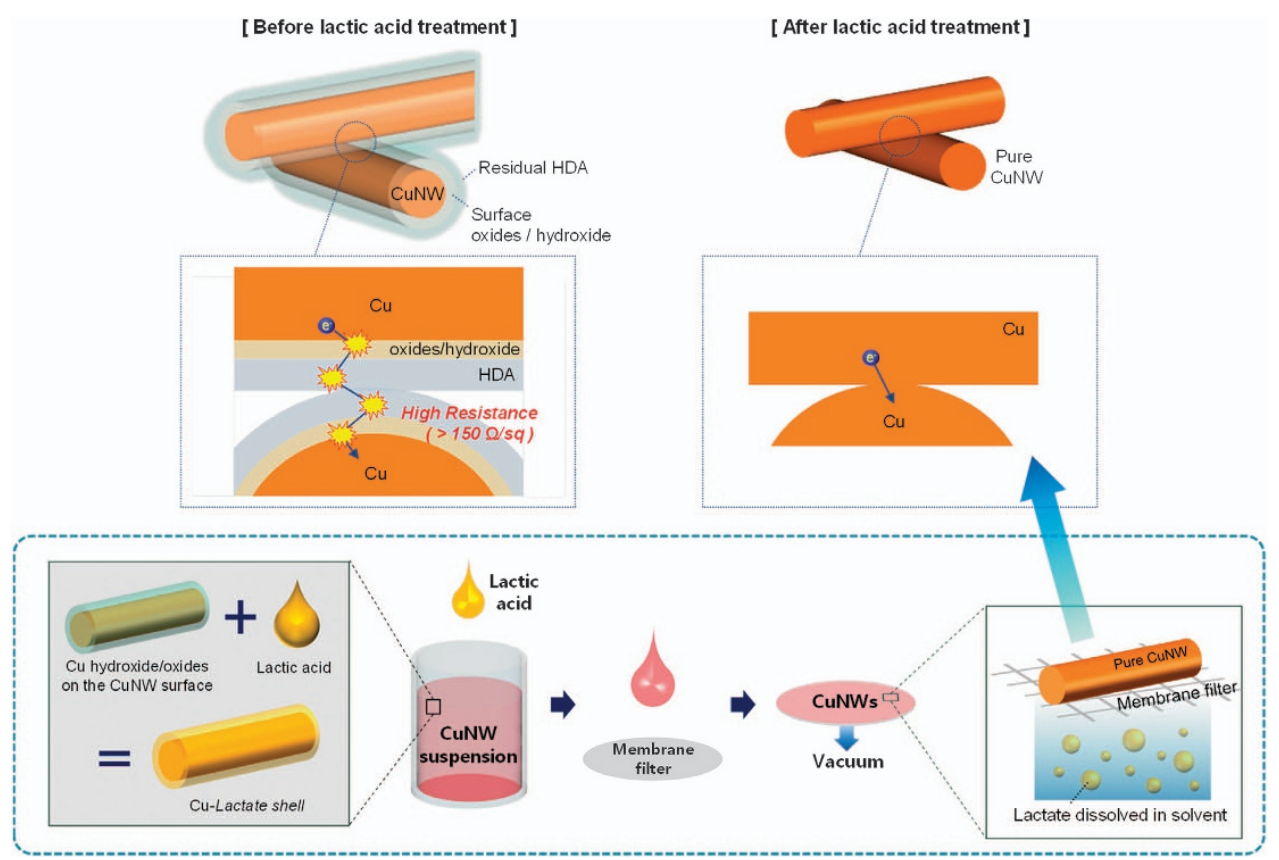

Figure 2 Schematic illustration of the lactic acid treatment process and the result. Before lactic acid treatment, the copper nanowires (CuNWs) were surrounded by residual hexadecylamine (HDA) and surface oxides/hydroxide, which hindered transference of electrons between nanowires. During the lactic acid treatment, these surface HDA and oxides/hydoxides were removed. After lactic acid treatment, the electrons can be transported between pure CuNWs.

A field emission scanning electron microscopy image showed a crosssectional view of the as-fabricated film; organic residues roughly surrounded the loosely-stacked nanowires (Figure 1a). The presence of organic materials was revealed by a HRTEM (Figure 1b). FT-IR spectroscopy was able to identify the organic phase adsorbed on CuNWs. The as-fabricated CuNW films showed a spectrum similar to HDA (Figure 3a). The peaks at $2917-2918 \mathrm{~cm}^{-1}$ and $2850 \mathrm{~cm}^{-1}$ were assigned to $\mathrm{C}-\mathrm{H}$ symmetric and asymmetric stretching modes, respectively. Unadsorbed HDA had a peak at $3332 \mathrm{~cm}^{-1}$ by the $\mathrm{N}-\mathrm{H}$ stretching mode. For the CuNW film, this peak was blue shifted by $90 \mathrm{~cm}^{-1}$ due to the chemisorbed amine group on copper. ${ }^{21-23}$ The peak assignments for FT-IR spectra are summarized in Supplementary Table S1. The as-synthesized CuNWs consisted of phase-pure FCC copper (JCPDS card no. 04-0836) as analyzed by X-ray diffraction (Supplementary Figure S1), while no oxide/hydroxide phases were detected. However, to acquire more accurate information on the presence of surface oxide/hydroxide, an XPS depth profile analysis was obtained (Figure $3 \mathrm{~b}$ ). The XPS spectral analysis in the $\mathrm{Cu} 2 \mathrm{p}_{3 / 2}$ region revealed the presence of $\mathrm{CuO}$ and $\mathrm{Cu}(\mathrm{OH})_{2}$ with binding energies of 933.2 and $934.8 \mathrm{eV}$, respectively. The main peak at $932.6 \mathrm{eV}$ corresponded to metallic copper. After argon ion etching for $60 \mathrm{~s}$, the peaks at 933.2 and $934.8 \mathrm{eV}$ disappeared, which implied that copper oxide/hydroxide, $2-12 \mathrm{~nm}$ in thickness, existed only on the surface. This provided an understanding of why as-synthesized CuNW films were highly resistive. The surface of CuNWs is oxidized during their synthesis in water according to the following equations, and the surface oxide/hydroxide hinders electron conduction between the neighboring nanowires.

$$
\begin{aligned}
& \mathrm{Cu}+1 / 2 \mathrm{O}_{2}+\mathrm{H}_{2} \mathrm{O} \rightarrow \mathrm{Cu}(\mathrm{OH})_{2} \\
& \mathrm{Cu}+1 / 2 \mathrm{O}_{2} \rightarrow \mathrm{CuO}
\end{aligned}
$$

Moreover, the residual HDA prevents the nanowires from forming firm contacts. It is difficult to completely remove the chemisorbed capping molecule, HDA, by a general washing technique. Post annealing at $250{ }^{\circ} \mathrm{C}$ under an inert atmosphere is generally required to remove HDA, by which the nanowire films become conductive. ${ }^{16}$

The CuNWs were chemically treated with lactic acid to completely remove both surface oxide/hydroxide and chemisorbed HDA. It has been reported that the lactic acid can react with copper oxide/ hydroxide to form an organic copper salt, which is subsequently reduced into elemental copper at elevated temperatures under a protective atmosphere. ${ }^{24,25}$ Therefore, as long as the resulting organic copper salt is soluble in a given solvent, lactic acid would be an effective chemical remover for both surface oxide/hydroxide. Lactic acid treatment leads to the formation of copper lactate as a result of the reaction with the copper oxide/hydroxide, as follows:

$$
\begin{aligned}
& \mathrm{Cu}(\mathrm{OH})_{2}+2\left[\mathrm{CH}_{3} \mathrm{CH}(\mathrm{OH}) \mathrm{COOH}\right] \\
& \rightarrow \mathrm{Cu}\left[\mathrm{CH}_{3} \mathrm{CH}(\mathrm{OH}) \mathrm{COO}\right]_{2}+2 \mathrm{H}_{2} \mathrm{O} \\
& \mathrm{CuO}+2\left[\mathrm{CH}_{3} \mathrm{CH}(\mathrm{OH}) \mathrm{COOH}\right] \\
& \rightarrow \mathrm{Cu}\left[\mathrm{CH}_{3} \mathrm{CH}(\mathrm{OH}) \mathrm{COO}\right]_{2}+\mathrm{H}_{2} \mathrm{O}
\end{aligned}
$$

Then, copper lactate, which is soluble in IPA, readily washes out, leaving behind the phase-pure CuNWs as shown in Figure 2. Before lactic acid treatment, the CuNWs were surrounded by residual HDA and surface oxides/hydroxide that hindered transference of electrons between nanowires. During the lactic acid treatment, these surface HDA and oxides/hydoxides were removed. After lactic acid treatment, the electrons can be transported between pure CuNWs. The surface etching was time dependent and was confirmed by the decreased nanowire diameter (Supplementary Figure S2). HDA was removed as the oxides were etched by the acid, because it was chemisorbed on the surfaces of oxide/hydroxide. The C 1s XPS result confirmed that the oxide/hydroxide disappeared as along with HDA after treatment with lactic acid (Supplementary Figure S3). The scanning electron microscope and transmission electron microscopy images also support the presumption that HDA is removed from the surface of the CuNWs by lactic acid treatment for $10 \mathrm{~s}$; the treated nanowires were densely packed to form a well-contacted network film (Figures 1c,d). In FT-IR 
and XPS analyses, peaks from HDA disappeared after lactic acid treatment and the copper lactate was invisible on the CuNWs, indicative of its complete dissolution in IPA (Figure 3a). Removal of the surface oxide/hydroxide was also demonstrated by XPS analysis. For the lactic acid-treated CuNWs, metallic copper was only observed by the absence of a peak at 933.2 and $934.8 \mathrm{eV}$ attributed to $\mathrm{CuO}$ and $\mathrm{Cu}(\mathrm{OH})_{2}$, respectively, regardless of the varying etching times (Figure $3 b$ ).

This chemical approach facilitates fabrication of a highly transparent and conductive electrode at room temperature without control of the atmosphere or temperature. Figure $3 \mathrm{c}$ shows a plot of the measured optical transmittance $(T)$ at $\lambda=550 \mathrm{~nm}$ versus $R_{\mathrm{sh}}$ for the lactic acid-treated CuNW films fabricated at $25^{\circ} \mathrm{C}$ in air. Some of the representative results reported in the literature for CuNW transparent conductors are marked in Figure $3 \mathrm{c}$. Both $R_{\mathrm{sh}}$ and $T$ decreased significantly with increasing nanowire density, which was controlled by the filtrate volumes of the CuNW dispersion with a concentration of $0.06 \mathrm{mg} \mathrm{ml}^{-1}$ (Supplementary Figure S4). These transparent electrodes exhibited the performance sufficient to be used as transparent conductors, with an $R_{\mathrm{sh}}$ of $19.8 \Omega \mathrm{sq}^{-1}$ and $T$ of $88.7 \%$. The nanowire density was calculated as $3.31 \times 10^{13}$ wires $\mathrm{m}^{-2}$ (Supplementary Figure S4 caption). The outstanding properties of this CuNW electrode were attributed to the high aspect ratio $(>750)$ of the nanowires as compared with others reported at 384 and 333. ${ }^{17,26-28}$ Chemical removal of the surface oxide/hydroxide and external pressured applied during the transfer process also allowed the CuNWs to make direct and tight contacts between pure CuNWs, leading to less junction resistance. ${ }^{29}$ If it is possible to fabricate the high aspect ratio and junction-less structured ${ }^{30}$ nanowire-based transparent conductors, it could be further meaningful result in terms of optical/electrical properties and the manufacturing cost.

Despite exceptional performance, bare CuNW films readily oxidize and suffer from poor adhesion to the substrate and low thermal stability in harsh environments. We recently reported a $\mathrm{ZnO} / \mathrm{AgNW} /$ $\mathrm{ZnO}$ composite electrode to resolve such issues. ${ }^{12}$ Similarly, we sandwiched the CuNWs with the sputtered AZO layers to form a composite structure. The outer layer of AZO-embedding in the CuNWs prevents the CuNWs from being oxidized or collapsed at elevated temperatures, facilitating antioxidation and structural stability. The HRTEM cross-sectional image revealed that the AZO (thickness ( $\mathrm{t}$ ):25 nm)/CuNW/AZO (t:25 nm) sandwich structure was perfectly formed with full coverage by an outer AZO layer (Figure 4a). The HRTEM image showed a cross-sectional view of lactic acidtreated CuNW, indicating that a fivefold twinned crystal structure is evident. Top and bottom AZO layers wrap around the CuNW without an air gap between the layers. Energy dispersive X-ray a

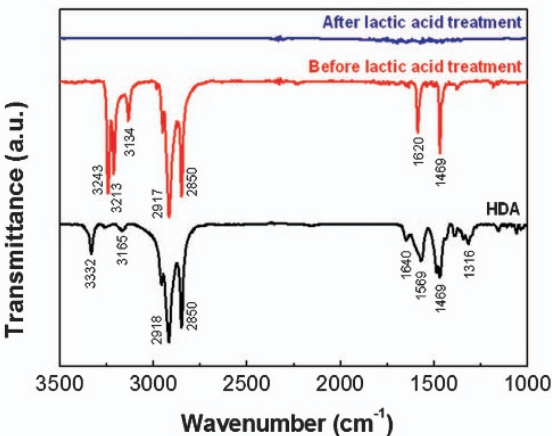

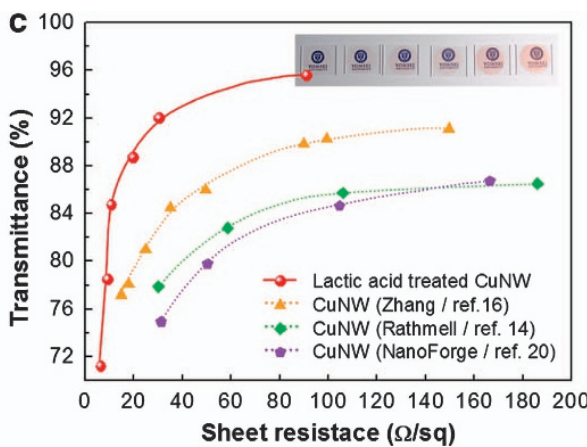

Sheet resistace $(\Omega / \mathbf{s q})$
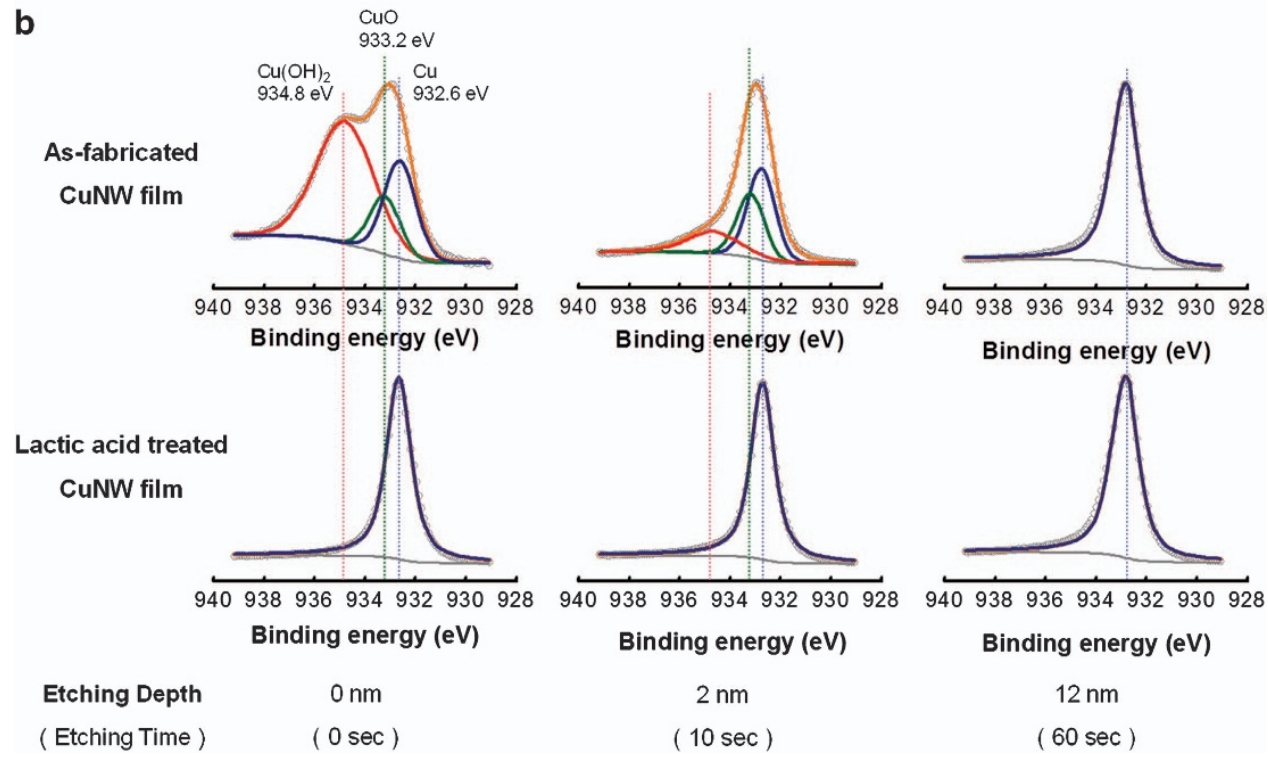

actic acid treated

( Etching Time)

$(0 \mathrm{sec})$

$(10 \mathrm{sec})$

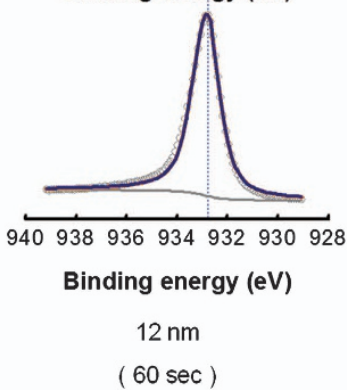

Figure 3 Influence of lactic acid treatment. (a) Fourier-transform infrared spectroscopy transmittance spectra for the hexadecylamine, as-fabricated copper nanowire (CuNW) film, and lactic acid-treated CuNW film. (b) $\mathrm{Cu} 2 \mathrm{p}_{3 / 2}$ X-ray photoelectron spectroscopy-depth profile of as-fabricated CuNW film and lactic acid-treated CuNW film. Ar-ion sputtering was performed from the surface as a function of etching time. (c) Plot of transmittance $(\lambda=550 \mathrm{~nm}) \mathrm{vs}$ sheet resistance for lactic acid-treated CuNW film, as compared to other references related to CuNW transparent electrodes. 

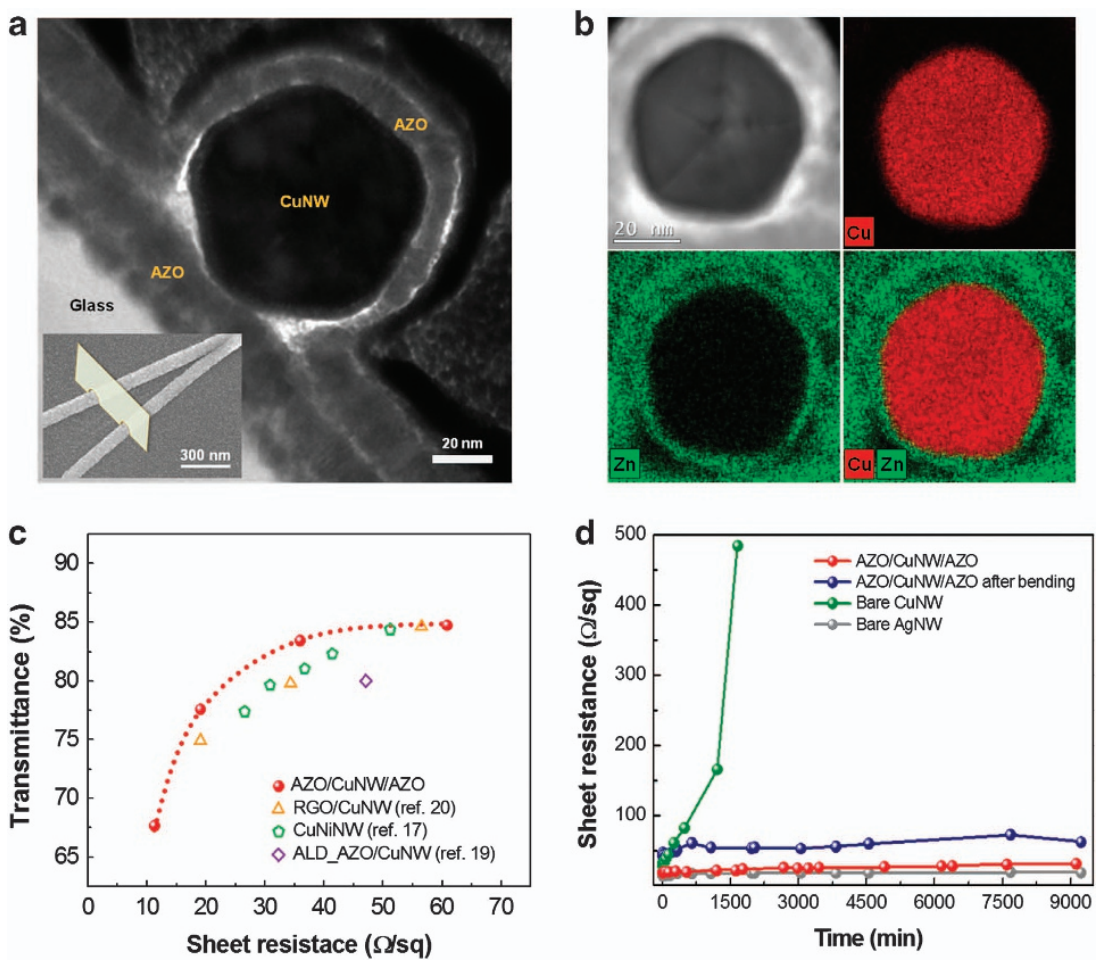

Figure 4 Oxidation stability of Al-doped ZnO (AZO)/CuNW/AZO composite electrode. (a) High-resolution transmission electron microscopy image of a crosssectioned AZO/CuNW/AZO composite. The inset scanning electron microscope image indicates the focused ion beam-assisted sectioning of the AZO/CuNW/ AZO composite. (b) Energy dispersive X-ray compositional mapping of the cross-sectioned AZO/CuNW/AZO composite shows a clear distribution of Cu and Zn components. (c) Plot of transmittance vs sheet resistance for AZO/CuNW/AZO composite, compared with other CuNW-based composite electrodes. (d) Variations of the sheet resistance at $80^{\circ} \mathrm{C}$ for CuNW single film, AgNW single film and AZO/CuNW/AZO film as a function of the exposure time. CuNW and AgNW film were fabricated on glass substrates, and AZO/CuNW/AZO film was prepared on both a glass and a polyethylene terephthalate (PET) substrate. The resistivity for PET substrate-based flexible AZO/CuNW/AZO films was analyzed after 1280 times bending cycles. For all composite electrode samples, the upper and bottom AZO layers have the same thickness of $55 \mathrm{~nm}$.

elemental mapping clearly demonstrated that the CuNW was well-encapsulated by an outer AZO layer, which is expected to provide the thermal stability of the composite electrode (Figure $4 \mathrm{~b}$ ). The thermal stability was improved by increasing the thickness of the outer AZO layer from $25-110 \mathrm{~nm}$, but a thicker layer caused transmittance loss (Supplementary Figure S5a); thus, we determined that a bottom and upper layer with the same thickness of $55 \mathrm{~nm}$ was the optimized structure of AZO/CuNW/AZO composite. The $R_{\text {sh }}$ and $T$ of the AZO/CuNW/AZO composite electrodes are shown in Figure 4c. When the $T$ was $83.94 \%$, the $R_{\mathrm{sh}}$ was $35.94 \Omega \mathrm{sq}^{-1}$. Even though the AZO/CuNW/AZO composite lost a transparency of $7 \%$ compared with the bare CuNW film, it was still superior to that reported in previous works. Transmittance of our composite electrode could be further enhanced by the process optimizations: the incorporation of CuNWs with high aspect ratio, the reduced CuNWs number density, the use of AZO layer with high transmittance and the thickness control of CuNWs network film and AZO bottom/top layers.

Moreover, the thermal resistance was greatly improved as the AZO upper layer thickness increased (Supplementary Figure S5b). The electrical properties of optimized composite electrode were maintained well up to $180^{\circ} \mathrm{C}$, which implies that both the thermal oxidation and the temperature-dependent structural collapse were effectively suppressed under temperatures at which the various flexible substrates, except for expensive, semitransparent polyimide substrate, are adoptable. This thermal stability is much superior to the previously reported CuNW-based transparent conductors. ${ }^{17,19,20}$
The sheet resistances of our composite electrodes showed nearly invariance during 5 months at room temperature (Supplementary Figure S6). Thus, we carried out the corresponding test under an accelerated oxidation condition at $80^{\circ} \mathrm{C}$ even if most of applications might be likely related to the room temperature operation for a prolonged time. Figure $4 \mathrm{~d}$ shows variations of $R_{\mathrm{sh}}$ as a function of the holding time at $80^{\circ} \mathrm{C}$ under an ambient atmosphere, a general condition for monitoring the thermal durability during device operations, for bare CuNW and AZO (55 nm)/CuNW/AZO (55 nm) composite electrodes. A lactic acid treatment was performed in both cases. The $R_{\mathrm{sh}}$ of the bare CuNW film rose dramatically at $80^{\circ} \mathrm{C}$ after $8 \mathrm{~h}$ due to the spontaneous oxidation of the CuNWs. On the other hand, the $R_{\mathrm{sh}}$ of the $\mathrm{AZO} / \mathrm{CuNW} / \mathrm{AZO}$ composite electrode was nearly constant (approximately doubled) at $80^{\circ} \mathrm{C}$ even after $166 \mathrm{~h}$ exposure, supporting the critical role of the thermally-stable outer AZO layer. After the thermal test at $80^{\circ} \mathrm{C}$, the shape of bare CuNWs changed, whereas the surface of the AZO/CuNW/AZO composite maintained its initial morphology (Figure 5).

In particular, the metal-nanowire network film exhibited its usefulness as a flexible transparent electrode. To ensure the reinforced anchoring between neighboring CuNWs during the bending test, the CuNW film transferred on polyethylene terephthalate substrate was post annealed at $180^{\circ} \mathrm{C}$ in a nitrogen atmosphere for $30 \mathrm{~min}$. Our CuNW films were prepared at room temperature, so that the individual nanowires could slide away under repeated bending stress. The bending test was performed with a bending radius of $2.5 \mathrm{~mm}$ (strain 1\%) (Figure 6a). Upon bending the ITO single layer film 

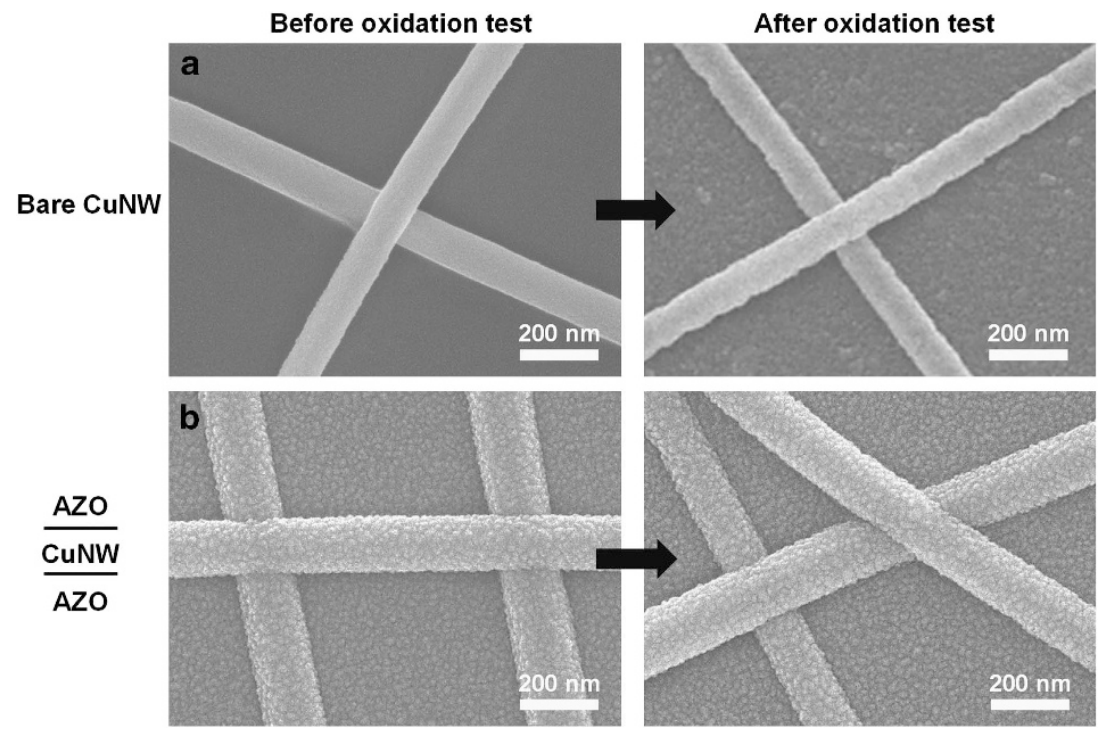

Figure 5 Scanning electron microscope images showing the morphological change of the nanowires before and after thermal oxidation tests under atmospheric conditions at $80^{\circ} \mathrm{C}$, for $2 \mathrm{~h}$. (a) Bare copper nanowire (CuNW) film and (b) Al-doped ZnO (AZO)/CuNW/AZO composite.
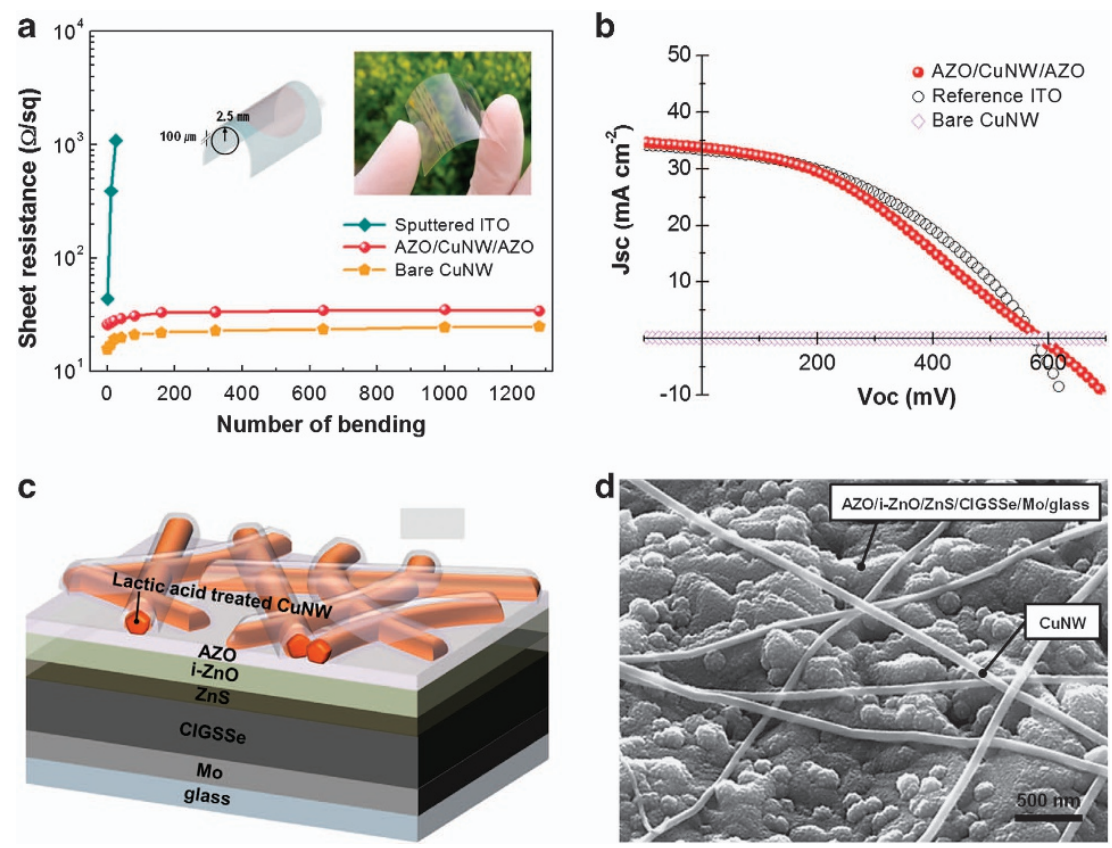

Figure 6 Al-doped ZnO (AZO)/copper nanowire (CuNW)/AZO composite electrode based thin-film solar cells. (a) Changes of the sheet resistance during the bend test for the sputtered indium tin oxide (ITO) single layer, CuNW single layer and AZO/CuNW/AZO composite layer on polymer substrates. Schematic showing the mechanical bend test conditions (left of inset) and a photograph of a flexible and transparent AZO/CuNW/AZO composite electrode on a flexible polymer substrate. (b) Photovoltaic $I-V$ characteristics displaying the power conversion efficiency of ITO single film, bare CuNW film and AZO/CuNW/AZO composite electrode-incorporated CIGSSe thin-film solar cells. (c) Schematic structure of CIGSSe thin-film solar cell using an AZO/CuNW/AZO composite electrode. (d) Scanning electron microscope image showing the surface of the CIGSSe thin-film solar cell using the AZO/CuNW/AZO composite electrode.

on a flexible substrate, the sheet resistance increased dramatically. In contrast, the AZO/CuNW/AZO composite electrode as well as bare CuNW film revealed good mechanical stability with a nearly constant sheet resistance even after 1280 bending cycles. The mechanical flexibility of our AZO/CuNW/AZO electrode results from unique composite structure in which flexible metal-nanowire networks are embedded between ultrathin inorganic metal oxide films. We observed the surface morphology of AZO/CuNW/AZO composite film fabricated on flexible substrate after a repeated bending test, as shown in Supplementary Figure S7. Any cracks were not detectable on the film, owing to the excellent mechanical property of ultrathin AZO layer. As additional evidence shown in Figure $4 \mathrm{~d}$, the sheet resistance of AZO/CuNW/AZO composite film on flexible substrate even after 1280 times bending cycles remained nearly constant during $166 \mathrm{~h}$ exposure to air at $80{ }^{\circ} \mathrm{C}$ (it should be noted that initial resistance slightly increased due to the repeated bending). If the AZO layer is damaged during the bending test, CuNWs would have been readily oxidized and their sheet resistance likely soars. These results clearly 
support that our AZO/CuNW/AZO composite film achieves not only the sufficient flexibility, but also the desired oxidation stability.

As a representative application example of a newly developed composite electrode, we exploited the successful implementation of the CuNW-based transparent electrode into $\mathrm{Cu}\left(\mathrm{In}_{1-x}, \mathrm{Ga}_{x}\right)(\mathrm{S}, \mathrm{Se})_{2}$ (CIGSSe) thin-film solar cells. The CuNW-based window electrodes were deposited on the intrinsic (i)-ZnO/ZnS(buffer layer)/CIGSSe (absorber layer)/Mo/glass to complete the solar cell fabrication. For the purpose of comparison, solar cells based on different transparent electrodes, such as ITO and bare CuNW, were produced. Except for the top transparent electrode, all layers in the devices were fabricated under identical process conditions. The schematic structure and scanning electron microscope image of the thin-film solar cell employing the $\mathrm{AZO} / \mathrm{CuNW} / \mathrm{AZO}$ electrode are shown in Figures $6 \mathrm{c}, \mathrm{d}$. Figure $6 \mathrm{~b}$ shows the photocurrent density-voltage $(J-E)$ curves of the CIGSSe thin-film solar cells under $100 \mathrm{~mW} \mathrm{~cm}^{-2}$ illumination (air mass $1.5 \mathrm{G}$ condition). The reference thin-film solar cell based on the sputtered ITO transparent electrode showed an open circuit voltage $\left(V_{O C}\right)$ of $574.4 \mathrm{mV}$, a short circuit current $\left(J_{S C}\right)$ of $33.4 \mathrm{~mA} \mathrm{~cm}^{-2}$, a fill factor $(F F)$ of $41.7 \%$ and calculated PCE of $7.9 \%$. These performance values are somewhat lower than the values reported for sputtered CIGSSe thin-film solar cells. This discrepancy resulted from the incorporation of a $\mathrm{ZnS}$ buffer layer instead of a CdS layer, which is well-known to outperform $\mathrm{ZnS}$, and the deposition conditions of the absorber and buffer layers were not yet optimized. However, the $\mathrm{ZnS} / \mathrm{CIGSSe} / \mathrm{Mo} /$ glass could serve as a platform by which to compare the influence of the top transparent electrodes.

Supplementary Table S2 summarizes the cell performance of the thin-film solar cells fabricated using different transparent electrodes. The thin-film solar cell employing single AZO layer (thickness= $110 \mathrm{~nm}$ ) deposited at room temperature without a post annealing exhibited the poor performance of $0.11 \%$ PCE because of the high

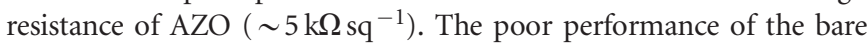
CuNW-based thin-film solar cell was caused by the unstable interface contact between rough $i-\mathrm{ZnO} / \mathrm{CIGSSe}$ layer and $\mathrm{CuNWs}$. In contrast, the facile insertion of a $\mathrm{CuNW}$ layer between the AZO double layers significantly improved cell performance, owing to the marked decrease in sheet resistance and resolved interface problem. The thin-film solar cell employing the AZO/CuNW/AZO exhibited a $V_{O C}$ of $585.8 \mathrm{mV}$, a $J_{S C}$ of $33.7 \mathrm{~mA} \mathrm{~cm}^{-2}$, a $F F$ of $36.0 \%$ and a calculated PCE of $7.1 \%$. Similar $V_{O C}, J_{S C}, F F$ and PCE values were observed when compared with the reference cell based on the ITO electrode. This observation supports the synergetic effect of the combination of AZO and CuNWs, which makes it possible to effectively transport the charge carriers to front contacts, reducing the current loss inside electrodes. The novelty of this study is the demonstration of the feasibility of noble metal-free, indium-free transparent electrodes that are comprised of only earth-abundant elements $(\mathrm{Cu}, \mathrm{Al}, \mathrm{Zn}$ and $\mathrm{O})$, by incorporating a new chemical approach to eliminate the surface impurities, organic moieties and oxide/hydroxide, as well as suggesting the thermally-resistive layered-structure against structural collapse and surface oxidation.

\section{CONCLUSIONS}

Here we fabricated stable AZO/CuNW/AZO composite electrodes by modulating the surface of the as-synthesized CuNWs with a simple chemical treatment using lactic acid. The highly transparent $(88.7 \%)$ and conductive $\left(19.8 \Omega \mathrm{sq}^{-1}\right)$ CuNW electrodes were prepared at room temperature without any atmospheric control. Through indepth studies based on XPS, transmission electron microscopy, scanning electron microscope and FT-IR analyses, it was revealed that lactic acid is a highly effective removal agent for residual organics and surface oxide/hydroxide, which influence the opto-electrical and structural properties of the CuNW network films. It was demonstrated that the lactic acid-treated CuNW network film, embedded in AZO layers, was an excellent transparent electrode material, which, so far, has performances superior to the reported CuNW-based transparent electrodes in terms of transmittance and sheet resistance $\left(35.9 \Omega \mathrm{sq}^{-1}\right.$ and $83.9 \%$ at $\left.550 \mathrm{~nm}\right)$. The mechanical flexibility on plastic substrates was robust due to the ductility and the onedimensional structure of CuNWs, and the thermal stability was dramatically improved owing the AZO layer fully covering the CuNWs. As a demonstration for potential use as a transparent electrode, the composite electrode developed in this study was used in CIGSSe thin-film solar cells, and the AZO/CuNW/AZO electrode showed PCE performances $(7.1 \%)$ comparable to conventional sputtered ITO-based solar cells.

\section{ACKNOWLEDGEMENTS}

This work was supported by a National Research Foundation (NRF) of Korea grant funded by the Korea government (MSIP; No. 2012R1A3A2026417) and the Basic Research Laboratory (BRL) Program (No. 2011-8-2048). It was also partially supported by the third Stage of Brain Korea 21 Plus Project.

1 Gordon, R. G. Criteria for choosing transparent conductors. MRS Bull. 25, 52-57 (2000)

2 Kim, M.-G., Kanatzidis, M. G., Facchetti, A. \& Marks, T. J. Low-temperature fabrication of high-performance metal oxide thin-film electronics via combustion processing. Nat. Mater. 10, 382-388 (2011).

3 Wu, Z., Chen, Z., Du, X., Logan, J. M., Sippel, J., Nikolou, M., Kamaras, K., Reynolds, J. R., Tanner, D. B., Hebard, A. F. \& A. G., Rinzler Transparent conductive carbon nanotube films. Science 305, 1273-1276 (2004)

4 Ratheesh Kumar, P. M. \& Vijayakumar, K. P. Spray pyrolysed zinc oxide thin films: effects of doping and ion beam irradiation, Ph.D Thesis, Cochin University of Science and Technology (2007).

5 Sánchez-Iglesias, A., Rivas-Murias, B., Grzelczak, M., Pérez-Juste, J., Liz-Marzán, L. M. Rivadulla, F. \& Correa-Duarte, M. A. Highly transparent and conductive films of densely aligned ultrathin Au nanowires monolayers. Nano Lett. 12, 6066-6070 (2012).

6 De, S. \& Coleman, J. N. The effects of percolation in nanostructured transparent conductors. MRS Bull. 36, 774-780 (2011).

$7 \mathrm{Hu}, \mathrm{L} ., \mathrm{Wu}, \mathrm{H}$. \& Cui, Y. Metal nanogrids, nanowires, and nanofibers for transparent electrodes. MRS Bull. 36, 760-765 (2011).

8 Lee, J.-Y., Connor, S. T., Cui, Y. \& Peumans, P. Solution-processed metal nanowire mesh transparent electrodes. Nano Lett. 8, 689-692 (2008).

9 Wu, H., Hu, L., Rowell, M. W., Kong, D., Cha, J. J., McDonough, J. R., Zhu, J., Yang, Y., McGehee, M. D. \& Cui, Y. Electrospun metal nanofiber webs as high-performance transparent electrode. Nano Lett. 10, 4242-4248 (2010).

10 Choi, K.-H., Kim, J., Noh, Y.-J., Na, S.-I. \& Kim, H.-K. Ag nanowire-embedded ITO films as a near-infrared transparent and flexible anode for flexible organic solar cells. Sol. Energ. Mat. Sol. C. 110, 147-153 (2013).

11 Chung, C.-H., Song, T.-B., Bob, B., Zhu, R. \& Yang, Y. Solution-processed flexible transparent conductors composed of silver nanowire networks embedded in indium tin oxide nanoparticle matrices. Nano Res. 5, 805-814 (2012).

$12 \mathrm{Kim}$, A., Won, Y., Woo, K., Kim, C.-H. \& Moon, J. A highly transparent low resistance $\mathrm{ZnO} / \mathrm{Ag}$ nanowire/ZnO composite electrode for thin film solar cells. ACS Nano 7 1081-1091 (2013).

13 Kang, M.-G., Park, H., Ahn, S. \& Guo, J. L. Transparent Cu nanowire mesh electrode on flexible substrates fabricated by transfer printing and its application in organic sola cells. Sol. Energ. Mat. Sol. C. 94, 1179-1184 (2010).

14 Rathmell, A. R. \& Wiley, B. J. The synthesis and coating of long, thin copper nanowires to make flexible, transparent conducting films on plastic substrates. Adv. Mater. 23, 4798-4803 (2011).

15 Jin, M., He, G., Zhang, H., Zeng, J., Xie, Z. \& Xia, Y. Shape-controlled synthesis of copper nanocrystals in an aqueous solution with glucose as a reducing agent and hexadecylamine as a capping agent. Angew. Chem. Inter. Ed. 50, 10560-10564 (2011).

16 Zhang, D., Wang, R., Wen, M., Weng, D., Cui, X., Sun, J., Li, H. \& Lu, Y. Synthesis of ultralong copper nanowires for high-performance transparent electrodes. J. Am. Chem. Soc. 134, 14283-14286 (2012).

17 Rathmell, A. R., Nguyen, M., Chi, M. \& Wiley, B. J. Synthesis of oxidation-resistant cupronickel nanowires for transparent conducting nanowire networks. Nano Lett. 12 3193-3199 (2012) 
18 Luo, X., Gelves, G. A., Sundararaj, U. \& Luo, J. L. Silver coated copper nanowires with improved anti-oxidation property as conductive fillers in low density polyethylene. Can. J. Chem. Eng. 91, 630-637 (2012).

19 Hsu, P., Wu, H., Carney, T. J., McDowell, M. T., Yang, Y., Garnett, E. C., Li, M., Hu, L. \& Cui, Y. Passivation coating on electrospun copper nanofibers for stable transparent electrodes. ACS Nano 6, 5150-5156 (2012).

20 Kholmanov, I. N., Domingues, S. H., Chou, H., Wang, X., Tan, C., Kim, J., Li, H., Piner, R., Zarbin, A. J. G. \& Ruoff, R. S. Reduced graphene oxide/copper nanowire hybrid films as high-performance transparent electrodes. ACS Nano 7, 1811-1816 (2013).

21 Korgel, B. A., Fullam, S., Connolly, S. \& Fitzmaurice, D. Assembly and selforganization of silver nanocrystal superlattices: ordered 'soft spheres'. J. Phys. Chem. B 102, 8379-8388 (1998).

22 Chen, M., Feng, Y., Wang, X., Li, T., Zhang, J. \& Qian, D. Silver nanoparticles capped by oleylamine: formation, growth, and self-organization. Langmuir 23, 5296-5304 (2007).

23 Porter, M. D., Bright, T. B., Allara, D. L. \& Chidsey, C. E. Spontaneously organized molecular assemblies. 4. Structural characterization of $n$-alkyl thiol monolayers on gold by optical ellipsometry, infrared spectroscopy, and electrochemistry. J. Am. Chem. Soc. 109, 3559-3568 (1987)

24 Deng, D., Cheng, Y., Jin, Y., Qi, T. \& Xiao, F. Antioxidative effect of lactic acid-stabilized copper nanoparticles prepared in aqueous solution. J. Mater. Chem. 22, 23989-23995 (2012).

25 Woo, K., Kim, Y., Lee, B., Kim, J. \& Moon, J. Effect of carboxylic acid on sintering of inkjetprinted copper nanoparticulate films. ACS Appl. Mater. Interfaces 3, 2377-2382 (2011).

26 Bergin, S. M. Chen, Y., Rathmell, A. R., Charbonneau, P., Li, Z. \& Wiley, B. J. The effect of nanowire length and diameter on the properties of transparent, conducting nanowire films. Nanoscale 4, 1996-2004 (2012).
27 Sorel, S., Lyons, P. E., De, S., Dickerson, J. C. \& Coleman, J. N. The dependence of the optoelectrical properties of silver nanowire networks on nanowire length and diameter. Nanotechnology 23, 185201 (2012).

28 Lee, P., Lee, J., Lee, H., Yeo, J., Hong, S., Nam, K. H., Lee, D., Lee, S. S. \& Ko, S. Highly stretchable and highly conductive metal electrode by very long metal nanowire percolation network. Adv. Mater. 24, 3326-3332 (2012).

29 Chen, R., Das, S. R., Jeong, C., Khan, M. R., Janes, D. B. \& Alam, M. A. Co percolating graphene wrapped silver nanowire network for high performance, highly stable, transparent conducting electrodes. Adv. Funct. Mater. 23, 5150-5158 (2013).

30 Wu, H., Kong, D., Ruan, Z., Hsu, P., Wang, S., Yu, Z., Carney, T. J., Hu, L., Fan, S. \& Cui, Y. A transparent electrode based on a metal nanotrough network. Nat. Nanotechnol. 8, 421-425 (2013).

(c) (1) () $\odot$ This work is licensed under a Creative Commons Attribution-NonCommercial-NoDerivs 3.0 Unported License. The images or other third party material in this article are included in the article's Creative Commons license, unless indicated otherwise in the credit line; if the material is not included under the Creative Commons license, users will need to obtain permission from the license holder to reproduce the material. To view a copy of this license, visit http://creativecommons.org/licenses/by-nc-nd/3.0/

Supplementary Information accompanies the paper on the NPG Asia Materials website (http://www.nature.com/am) 